\title{
Synbiotic supplementation with prebiotic Schizophyllum commune derived $\beta-(1,3 / 1,6)$-glucan and probiotic concoction benefits gut microbiota and its associated metabolic activities
}

Vineet Singh ${ }^{1 \dagger}$, Karthika Muthuramalingam ${ }^{2+}$, Young Mee Kim², Sanggyu Park ${ }^{3}$, Sung Hong Kim ${ }^{4}$, Jongdae Lee ${ }^{4}$, Changlim Hyun ${ }^{5}$, Tatsuya Unno ${ }^{1,6^{*}}$ and Moonjae Cho $2,7,8^{*}$

\begin{abstract}
Synbiotics synergistically favors beneficial effects of prebiotics and probiotics towards host metabolic health by modulating gut ecosystem. In this study, we sought to examine the effects of prebiotics (Schizophyllum commune derived $\beta-(1,3 / 1,6)$-glucan), probiotics (concoction made of eight different bacterial strains) and synbiotics (prebiotics + probiotics) on gut microbiota and its associated metabolic functions through $16 \mathrm{~S}$ rRNA gene sequences analysis. Results showed that probiotics strains used in this study were detected more in the synbiotic and probiotic treatments, while prebiotic dietary intervention increased the total bacterial abundance and metabolisms related to host immune strengthening. Probiotics and synbiotics dietary interventions enhanced similar metabolisms relating to butanediol and s-adenosyl-L-methionine biosynthesis. Probiotics treatment also showed depleted metabolic activities related to SCFA productions, that were not depleted in prebiotics treatment. With varying differential abundance patterns and metabolic activities across the treatments, our results suggest that synbiotic treatment provide more beneficial effects over probiotics and prebiotics.
\end{abstract}

Keywords: Synbiotics, $\beta-(1,3 / 1,6)-G l u c a n$, Gut microbiota, Butanediol biosynthesis, Adenosyl-L-methionine biosynthesis

\section{Introduction}

Gut microbiota, the virtual metabolic 'organ' consisting of intricate microbial ecosystem inhabiting gastrointestinal tract (GIT), has predominant and diverse effects on the traits of human health relating to physiology,

\footnotetext{
*Correspondence: tatsu@jejunu.ac.kr; moonjcho@jejunu.ac.kr

${ }^{\dagger}$ Vineet Singh and Karthika Muthuramalingam contributed equally to this work

${ }^{1}$ Faculty of Biotechnology, School of Life Sciences, SARI, Jeju National University, Jeju 63243, Republic of Korea

2 Department of Biochemistry, School of Medicine, Jeju National University, Jeju 63243, Republic of Korea

Full list of author information is available at the end of the article
}

immunology, neurology and metabolomics [1, 2]. Any disruption/imbalance in the gut microbial complex has been pathologically linked with non-infectious chronic disorders such as obesity, metabolic syndrome, neurodegenerative conditions, hypertension, hypercholesteremia, cancer, diabetes, chronic inflammatory diseases such as inflammatory bowel syndrome (IBD), Crohn's disease etc. [3, 4]. Besides host genetics and heritability, several external factors such as food, environment, medications, age, stress etc., is said to influence the host gut microbial composition [5-7]. Among these factors, the contribution of diet towards gut and systemic health is highly 
considered for a therapeutic strategy in manipulating the host gut microbiome [8-10].

Dietary prebiotics, the non-digestible food ingredients confer beneficiary effects on host health by supporting growth of probiotics, the live microorganisms that could restore and/or improve gut flora [7]. As the digestive/ absorptive resistance prebiotics enters from the small intestine into the colon, gut microbes start fermenting them thereby producing nutrients and other necessary substances such as antibiotics, anti-carcinogens, inhibitors of bacterial toxin production etc. [11]. In order for the probiotics to exhibit its utmost potential, they should be accompanied with appropriate prebiotics-collectively termed as 'synbiotics' [12].

Synbiotics with multi-strain bioactive probiotics ( $L$. plantarum, L. fermentum and B. infantis) and Triphala, a polyphenol-rich prebiotics enhanced the motility and fitness of Drosophila melanogaster in-addition to imposing beneficial effect on gut microflora in a simulated model of the human GIT [13]. Synbiotic administration of L. plantarum and inulin improved cognitive response associated gut microbial composition in Type 2 diabetes mellitus metabolic condition compared to their individual counterpart [14]. Long-term dietary intervention using the synbiotic formulation consisting of cauliflower mushroom derived $\beta$-glucan and L. fermentum alleviated estrogen-deficiency associated metabolic disturbances in ovariectomized rat models. It also reverses the imbalance created in the Firmicutes:Bacteroidetes ratio [15]. In another study, dietary intervention using synbiotic formulation consisting of probiotics such as $B$. animalis and $L$. paracase $i$ together with $\beta$-glucan, the oats-derived dietary fiber in male C57BL/6J mice models attenuated high fat diet (HFD) induced obesity and other metabolic complications [16]. Further, this intervention significantly restored the gut homeostasis at the phylogenetic and functional level in terms of richness and abundance of the gut microflora, microbial metabolites such as short chain fatty acids (SCFA) and bile acids (BA), metaproteomic pathways etc. Thus, with deliberate selection of pertinent prebiotics, the functionality of probiotics can be enhanced aiding synergistic effect on desirable health conditions and re-establishment of gut homeostasis.

Herein this study, we investigated the synbiotic impact of $S$. commune derived dietary prebiotic $\beta-(1,3 / 1,6)$ glucan and probiotic concoction consisting eight bacterial strains towards host gut microbiota and its associated metabolic pathways.

\section{Materials and methods}

Description of prebiotic, probiotic and synbiotic feed used

Prebiotics: $1 \% \beta-(1,3 / 1,6)$-glucan isolated as exopolysaccharide upon fermenting Schizophyllum commune
(Quegen Biotech Co. Ltd. Republic of Korea) with a concentration of $3 \mathrm{~g}$ per kilogram of normal diet feed. Probiotics: concoction (with a concentration of $15 \mathrm{~g}$ per kilogram of normal diet feed) containing eight different bacterial strains. The probiotics was formulated and procured from CKD Bio, Seoul, Republic of Korea. Dietary supplement with probiotic concoction containing Bifidobacterium, Lactobacilli and Streptococcus thermophiles were chosen, as they have the ability to re-configure gut microbiota with beneficial microbes. The characteristics of prebiotic fiber and probiotic bacterial loads were described in Fig. 1. Synbiotics: blend of prebiotics and probiotics in the normal diet feed at concentrations indicated in Fig. 1.

\section{Animal model}

Five-weeks old ICR mice (a general-purpose mouse model) were used in this study. Experimentation involving animals were ethically approved and were in accordance with the guidelines framed by Animal care and Use Committee (ACUC No. 2019-0019), Jeju National University. With an adaptation period of 2-weeks to our laboratory ambience, the mice were randomly sorted into four groups with $n=6$ per group as follows: normal diet, prebiotic diet, probiotic diet and synbiotic diet. Ad libitum supply of food and water was provided to the mice at all time-points of the experiment. Food, water and mice bedding in the cages were changed twice a week during the entire experimental time scale of 5 weeks.

\section{Experimental design}

The experiment was carried out as depicted in Additional file 1: Figure S1. At the end of the experimental duration, mice were sacrificed through inhalation anaesthetics. A day prior to the sacrifice, fecal samples collected from individual mice were taken for the analysis of fecal microbiota.

\section{Histology}

Upon sacrificing the mice, intestinal samples were collected and fixed in formalin solution. Tissue processing was carried out using alcohol/xylene solution followed by embedding them in hot paraffin wax. Using microtome, intestinal tissue was sectioned and stained using Haematoxylin-Eosin-method. Microscopic analysis of the stained section was done using Olympus BX51 microscope at the indicated magnification scale. Thickness of mucosal and sub-mucosal layer of the intestine tissue was quantified using ImageJ software.

\section{Microbial community analysis}

Fecal DNA was obtained using PowerFecal DNA extraction kit (QIAGEN, Germany). V4 region of 16S rRNA 

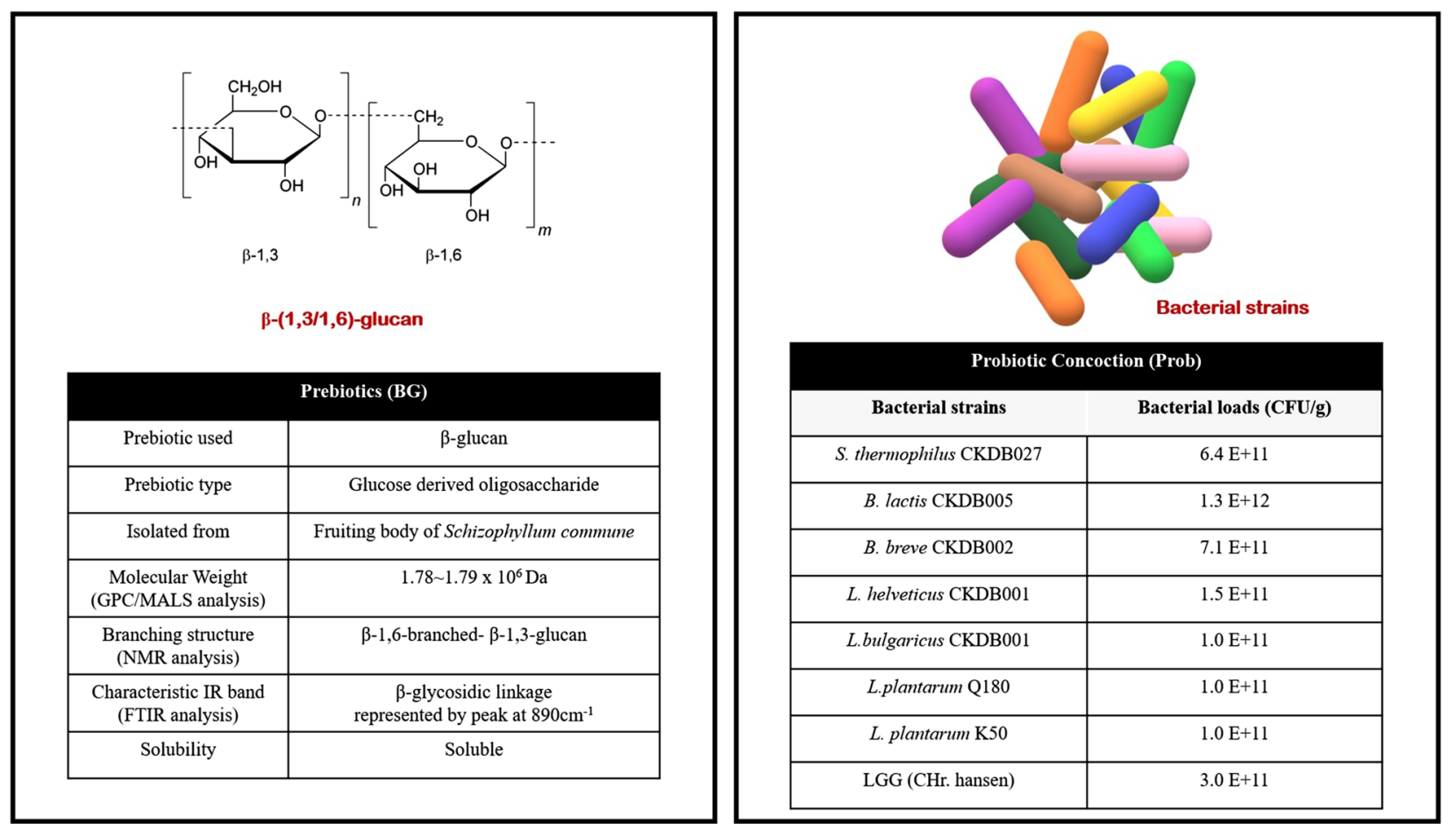

Fig. 1 Description of prebiotics and probiotics. GPC gel permeation chromatography, MALS multi-angle light scattering, NMR nuclear magnetic resonance, FTIR Fourier transform infra-red, CFU colony-forming units

gene was amplified using 515F (5'-GTGCCAGCMGCC GCGGTAA-3') and 806R (5'-GGACTACHVGGGTWT CTAAT-3') primer set. Two-step PCR was done to prepare MiSeq library and sequenced at Macrogen (Seoul, South Korea) according to the manufacture's instruction. Sequence data were processed using MOTHUR [17] according to the standard operational protocol (https:// mothur.org/wiki/miseq_sop/) with a slight modification of adding singleton removal after pre.cluster [18] subroutine. Silva.nr_v132 [19] was used for alignment and RDP version 11.5 [20] was used for taxonomic classification. Operational taxonomic units (OTUs) were assigned using opti.clust algorithm [21] with a sequence distance 0.03 .

MOTHUR was used for calculating ecological indices (Chao and Shannon) and non-metric multidimensional scaling (NMDS). NMDS was plotted using vegan R package to draw ellipse with $95 \%$ confidence level. PICRUSt2 $[22,23]$ was used to predict metabolic activities based on $16 \mathrm{~S}$ rRNA gene sequences.

\section{Statistical analysis}

Results of histology analysis was given as average \pm standard error and significance was observed using student $\mathrm{t}$-test method with $\mathrm{p}<0.05$ considered to be statistically significant. MOTHUR was used to conduct analysis of molecular variance (AMOVA) to examine significant difference between groups on NMDS plots. Differential abundance analysis was performed using LEfSe [24] and ALDEx2 [25] for OTUs and predicted metabolic activities, respectively. Spearman correlation analysis was performed to identify associations between differentially abundant OTUs and predicted metabolic activities. ANOVA was applied to compare ecological indices.

\section{Results}

Effects of treatments on gut architecture

Mice groups fed with prebiotics, probiotics and synbiotics does not have any dysbiosis in the mucosal as well as muscularis propria layer (Fig. 2). A statistically significant increase in the thickness of mucosal and muscularis propria was observed in the mice group fed with prebiotics, probiotics and synbiotics when compared to that of the normal diet group.

\section{Effects of treatments on microbial communities}

Good's coverage obtained for sequenced data showed greater than 99\% (Additional file 2: Figure S2), indicating enough sequencing depth was achieved in this study. Our results from Additional file 3: Figure S3 showed that there was not significant difference among the treatments in the microbial composition at the phylum (Additional 

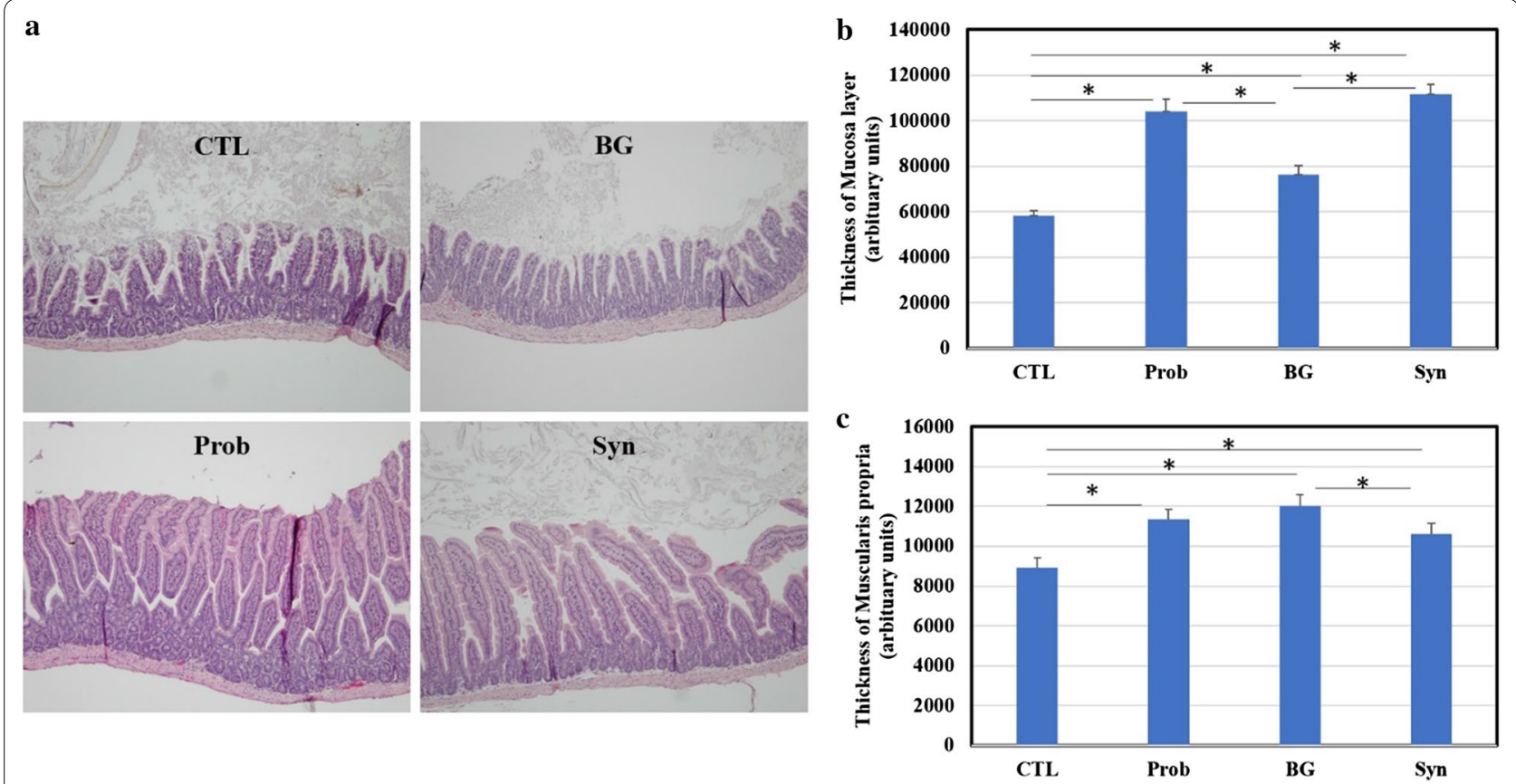

Fig. 2 Effect of prebiotics, probiotics and synbiotics on intestinal physiology: a microscopic observation (@ ×10 magnification) of H\&E stained intestinal sample isolated from mice fed with different diets; thickness of $\mathbf{b}$ mucosa and $\mathbf{c}$ muscularis propria layer of the intestine sample quantified using ImageJ software. * Corresponds to $\mathrm{p}<0.05$ (using student t-test) and is statistically significant

file 3: Figure S3A), family (Additional file 3: Figure S3B), and genus level (Additional file 3: Figure S3C). All the samples showed higher occurrence of Firmicutes, Bacteroidetes and Actinobacteria at the phylum level, and members of Porphyromonadaceae and Lachnospiraceae, Lactobacillaceae, and Bacteroidaceae were prominent at the family level. Accordingly, Lactobacillus and Bacteroides and unclassified genera of those families showed higher abundance compared to the other genera.
Ecological indices have shown a significant rise in microbial richness (chao) after beta glucan $(B G)$ treatment against control $(\mathrm{p}<0.05)$ and synbiotics $(\mathrm{p}<0.05)$, although microbial diversity (Shannon) remained unaffected in all the administered treatments (Fig. 3). $\beta$-Diversity analysis has shown distinct distribution for different groups, though probiotic and synbiotic sample groups were quite similar as shown by non-metric multidisciplinary scaling (NMDS) (Fig. 4). Analysis of $\mathbf{a}$

a

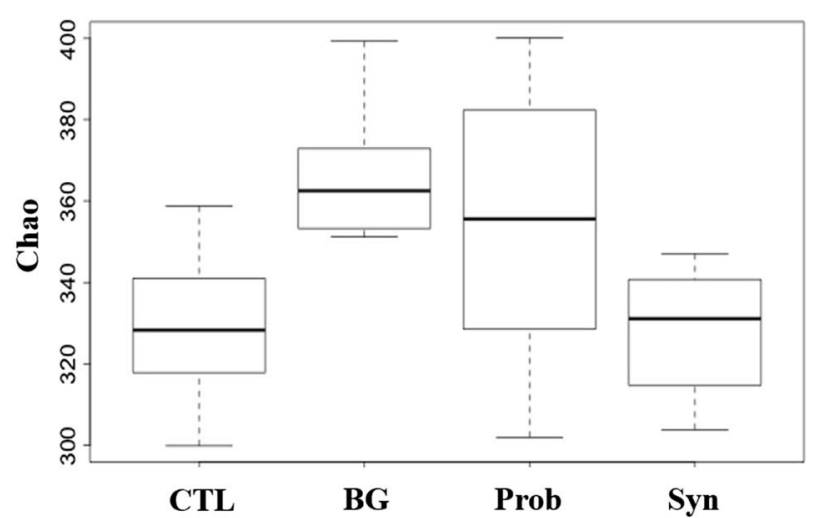

b

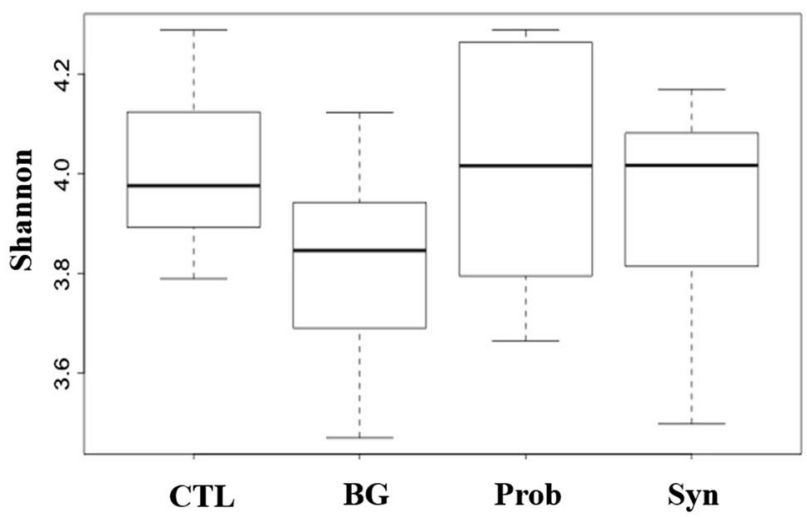

Fig. 3 Analysis of species richness (a) and evenness (b). ${ }^{*}$ Indicate significant difference $(p<0.05)$ 


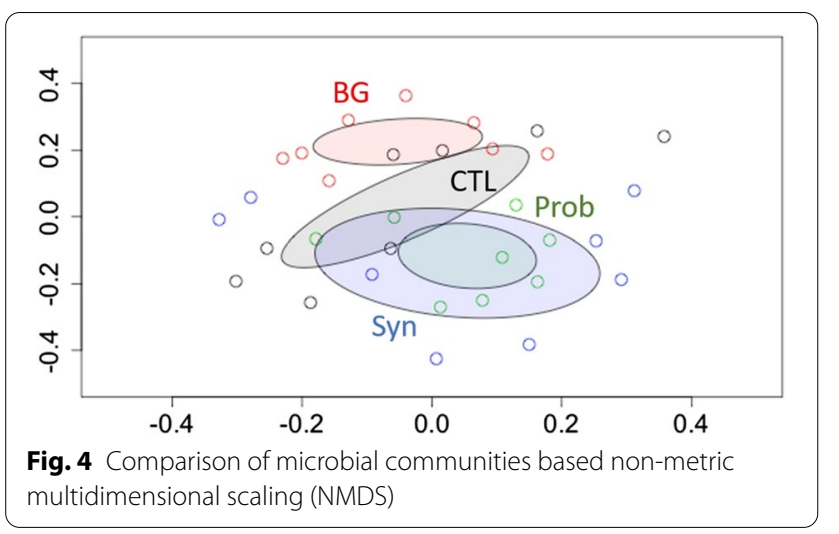

Table 1 Analysis of molecular variance (AMOVA)

\begin{tabular}{llll}
\hline Fs & CTL & BG & Prob \\
\hline CTL & & & \\
BG & 1.45 & & \\
Prob & $2.38^{* *}$ & $4.42^{* * *}$ & \\
Syn & 1.79 & $3.65^{* * *}$ & 1.50 \\
\hline
\end{tabular}

${ }^{*} p<0.05 ;{ }^{* *} p<0.01$ and ${ }^{* * *} p<0.001$

molecular variance (AMOVA) also had shown a significant difference in diversity induced by BG treatment against probiotic $(\mathrm{p}<0.001)$ and synbiotic $(\mathrm{p}<0.001)$ (Table 1).

Differentially abundant OTUs were identified using LEfSe and summarized in Fig. 5. Results in Fig. 5 showed that probiotic and synbiotic treatment significantly increased Bifidobacterium, Streptococcus, Lactobacillus and unclassified species of the family Porphyromonadaceae. BG treatment increased unclassified member of the family Lachnospiraceae, while probiotic treatment increased the genera Atopostipes, Corynebacterium, Jeotgalicoccus and Aerococcus. Distinctly, synbiotic treatment increased Parabacteroides. On the other hand, all the treatments showed a decrease of diverse unclassified members of Lachnospiraceae and Porphyromonadaceae.

\section{Effects of treatments on predicted intestinal metabolic functions}

PICRUSt 2 was used to predict metabolic activities based on microbial community. We have applied differential abundance comparison using ALDEx2 to identify significantly enriched or depleted metabolic activities. Results in Fig. 6 show that BG have shown enriched metabolic activities related with biosynthesis of nucleobases, mixed acid fermentation and colanic acid, whereas synbiotic and probiotic treatments enriched metabolic activities related to butane diol and methionine biosynthesis

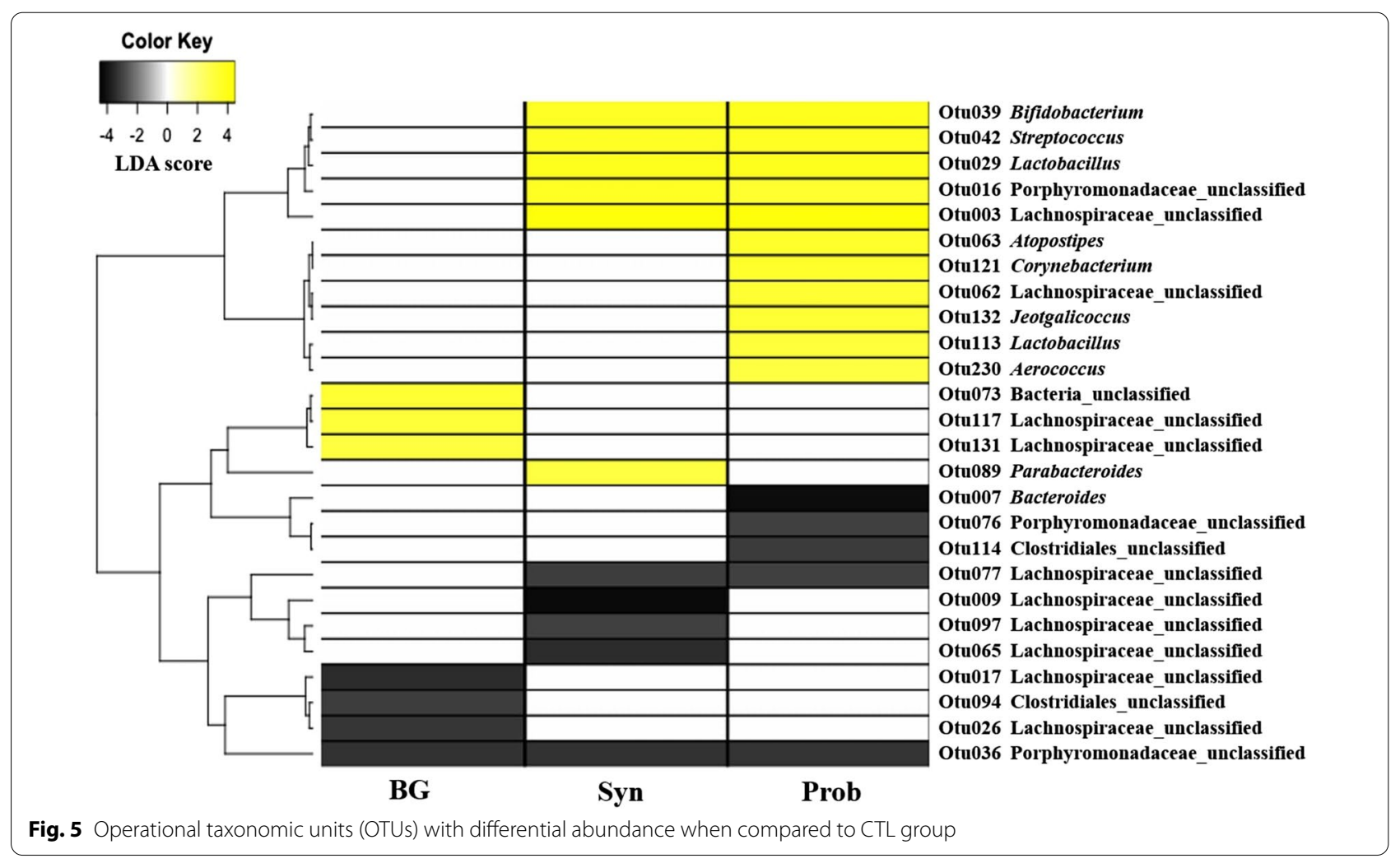



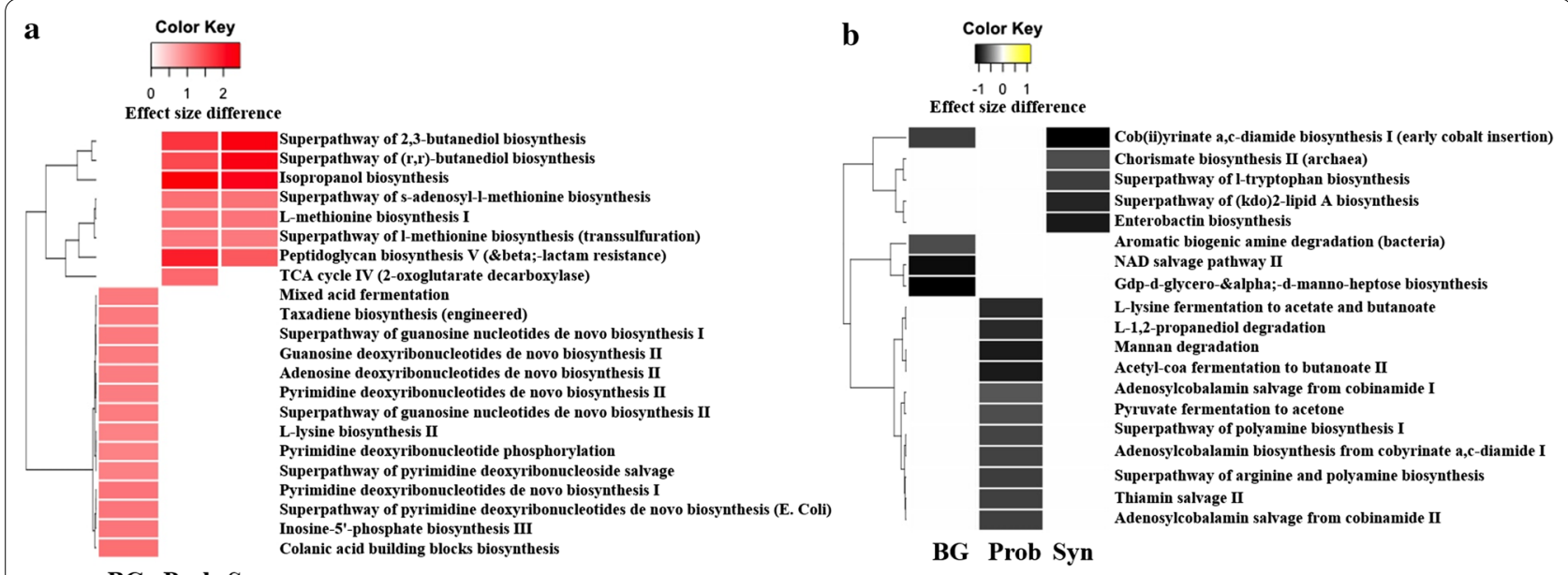

BG Prob Syn

Fig. 6 Significantly enriched (a) and depleted (b) metabolic activities predicted based on 16S rRNA gene sequence abundance

(Fig. 6a). In addition, probiotic treatment also increased the 2-oxoglutarate decarboxylase biosynthesis (Fig. 6a).

PICRUSt analysis also showed that probiotics treatment significantly decreased various metabolic activities where each treatment showed unique patterns (Fig. 6b). Our results suggest that synbiotic treatment decreased metabolic activities of biosynthesis of chorismite, tryptophan and enterobactin, BG decreased aromatic biogenic amine degradation, NAD salvage pathway II and mannohepatose biosynthesis and a number of metabolisms was depleted by probiotics treatment, including fermentation for SCFA production, mannan degradation, biosynthesis of polyamine and adenosylcobalamin (vitamin B12) production.

\section{Associated bacteria with the significantly altered pathways} Spearmen correlation analysis have calculated positive correlations between significantly $(\mathrm{p}<0.05)$ enriched de novo biosynthesis pathways of nucleobases (i.e. adenosine, guanosine and pyrimidine deoxynucleotide) with Lachnospiraceae_unclassified (OTU003), Lactobacillus (OTU029, 113), Streptococcus (OTU042), Bifidobacterium (OTU039), and Corneybacterium (OTU121). In addition, metabolisms related with $2-3$ butanediol synthesis and mixed acid fermentation were also found positively correlated with Clostridiales_unclassified (OTU114) and Lachnospiraceae_unclassified (OTU009, 065). Negative correlations were also observed between Lachnospiraceae_unclassified (OTU009) and biosynthesis of nucleobases. Likewise, the abundance of Parabacteroides (OTU089), Lachnospiraceae_unclassified (OTU003), Lactobacillus (OTU029, 113) and Streptococcus (OTU042) were negatively correlated with the mixed acid fermentation and 2-3 butanediol biosynthesis (Fig. 7a).

On the other hand, association with differentially abundant bacteria and significantly $(p<0.05)$ depleted metabolisms were investigated (Fig. 7b). Decrease in aromatic biogenic amine degradation and tryptophan synthesis was positively correlated with the Porphyromonadaceae_unclassified (OTU016) and Atopostipes (OTU063). Similarly, adenosylcobalamine salvage from cobinamide-II was associated with the Lactobacillus (OTU029, 113), Streptococcus (OTU042), Bifidobacterium (OTU039), while adenosylcobalamine salvage from cobinamide-I was found to be positively correlated with the Clostridiales_unclassified (OTU114) and Lachnospiraceae_unclassified (OTU009). Additionally, decrease in enterobactin and adenosylcobalamine salvage from cobinamide-I were negatively correlated with the $\mathrm{Lac}$ tobacillus (OTU029, 113), Streptococcus (OTU042) and Lachnospiraceae_unclassified (OTU003) (Fig. 7b).

\section{Discussion}

Gastrointestinal (GI) tract organs such as stomach, small intestine and colon along with other accessory organs such as liver, pancreas etc., are involved in the functions of digestive system such as breakdown of foods, nutrient absorption and excretion of solid waste apart from housing trillions of microorganisms with proper barrier levels [26]. Cross-section of GI tract in the intestinal organ consists of mucosa, submucosa, muscularis propria, serosa, mesentery etc. from the lumen to the outer wall. Mucosa, the innermost layer, consists of epithelial layer, lamina propria and muscularis mucosa. Organised into crypts and villi, the single, continuous epithelium layer contains diverse cells such as enterocytes, Paneth cells, goblet cells 

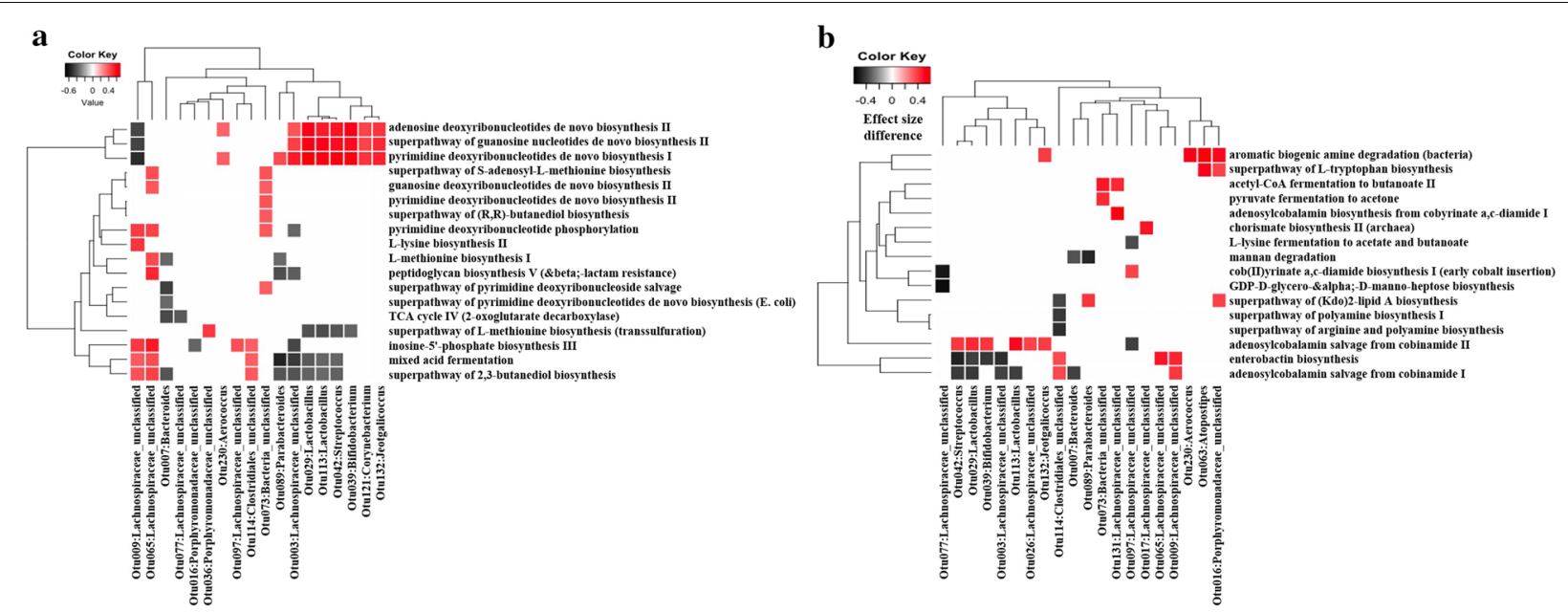

Fig. 7 OTUs associated with significantly increased (a) and decreased (b) metabolisms by treatments $(p<0.01)$

etc. Among these cell types, goblet cells secrete mucin, the heavily glycosylated proteins involved in the first line of defence against gut microbial encroachment into the intestinal wall. Muscularis propria is in charge of the peristaltic propulsion of food through the intestine, the defects of which leads to intestinal motility disorders and obstruction disorders [27, 28]. Thus, the architecture conservation of the GI tract compartments is of prime importance. From Fig. 2, it was observed that prebiotics, probiotics and synbiotics used in this study conserved the intestinal architecture and even resulted with increased thickness of mucosal and muscularis propria layer thickness, thereby attenuating gut motility disorders, and defending microbial encroachment into intestinal walls.

Microbial ecology among different treatment have shown that effects of probiotics and synbiotics were not of much difference (Fig. 3a, b), but microbial richness was significantly increased after BG treatment, as BG is a fine prebiotic and was expected to be utilized as carbon source by diverse microbes [29]. According to the differential abundance tests, our results showed only synbiotics and probiotics groups increased the abundance of numerous beneficial microbes i.e. Bifidobacterium, Streptococcus, Lactobacillus, and Lachnospiraceae [30-32]. In addition, synbiotic treatment also increased the abundance of Parabacteroides (OTU089) and had higher count of unclassified species of the Clostridiales (OTU114), and Bacteroides (OTU007), compared to probiotic treatment. Some of the Clostridiales have been reported to be butyrate producers [33]. Some species of Parabacteroides and Bacteroides have been recently reported to be protective against inflammation in the gut such as IBS [34]. Bacteroides has been also reported to benefit their host by excluding pathogens to colonize in the gut [35]. These beneficial gut microbes were not increased by BG treatment.

We found that BG treatment mostly enhanced the metabolisms related to biosynthesis of nucleotides (Fig. 6a). Perhaps, there may have been an increase in absolute abundance of intestinal bacteria. It has been reported that the use of beta-glucan supplement increased number of probiotics strains, which leads to improvement of intestinal flora [36]. However, in this study, beta-glucan was treated to mice where gut dysbiosis was not induced. Therefore, our results could be expected. Colanic acid was also increased by BG. It has been reported that colanic acid promotes mitochondrial fission and enhances the mitochondrial unfolded protein response under stress conditions, suggesting that anti-aging potentials in the BG treatment [37]. On the other hand, metabolic prediction analysis showed quite a similar response for probiotic and synbiotic treatment, which includes biosynthesis of methionine and peptidoglycan. It has been reported that 2-oxoglutarate decarboxylase provides succinylCoA for methionine biosynthesis, which leads to peptidoglycan biosynthesis [38], therefore, these enriched metabolic activities are related to each other. Methionine is an important sulfur containing amino acid which have nutritional and structural importance and simultaneously it is also the precursor of the enzyme $s$-adenosyl-L-methionine [39]. Enzyme $s$-adenosylL-methionine have numerous role including liver protection against the reactive oxygen species generated from the pathogens and alcohol consumption [39] and catalyzes trans-sulfuration reaction which is important for membranous structure and functions [39]. Butanediol biosynthesis was also enriched by probiotics and 
synbiotics, suggesting that extended fermentation of pyruvate in gut and possible anti-obesity effects [40] (Fig. 6a).

PICRUSt 2 analysis also revealed depletion in large number of beneficial metabolisms after probiotic treatments which includes adenosylcobalamin biosynthesis, polyamines and butyric acid (Fig. 6b). Butyric acid is perhaps most well-studied beneficial products in gut, while adenosylcobalamin, also known as vitamin B12, and polyamines have been reported as key modulators contributing in the structure and function of human gut microbial communities $[41,42]$. Therefore, the use of probiotics may have unbeneficial side effects. In contrast, synbiotic treatment did not show decrease in these metabolic activities but showed decrease in enterobactin and tryptophan synthesis. Tryptophan biosynthesis is a feedback inhibition process and it has been reported increased superpathway of L-tryptophan biosynthesis did not increase the amount of serum tryptophan [43]. Enterobactin is required by microbes for iron acquisition [44] and $\mathrm{Cob}(\mathrm{II})$ yrinate a,c-diamide biosynthesis I is involved in vitamin B12 synthesis using cobalt. Depletion of these could be caused by beta-glucan as they are known to bind to metal ions [45]. BG treatment also depleted aromatic biogenic amine degradation, NAD salvage pathway II and manno-heptose biosynthesis. Biogenic amines in the gut are important metabolites that play a crucial role in the regulation of intestinal functions such as digestion, absorption and immunity [46]. Depletion of the biogenic degradation may increase the availability of biogenic amines. Moreover, blocking NAD metabolisms has been reported to limit intestinal inflammation [47]. To support this, manno-heptose has been reported to increase under intestinal inflammation [48]. Therefore, BG treatment alone may increase host immunity. Together, the differential abundance of the predicted metabolic activities indicated unique metabolic consequences among the treatments: BG showing increased bacterial abundance and immunity; probiotics and synbiotics showing increased butanediol and $s$-adenosyl-L-methionine contributing to beneficial effects such as anti-obesity and liver protection. Probiotics, however, seems to deplete some of the metabolic activities related to vitamin B12 biosynthesis, which was not the case for synbiotics.

Correlation analysis between abundance of bacteria and metabolic predictions indicate association of taxa with metabolic activities. Our results showed enriched nucleobase biosynthesis were mostly associated with probiotic strains such as Lactobacillus, Bifidobacterium and Streptococcus, which were negatively associated with methionine biosynthesis, acid fermentation and butanediol biosynthesis (Fig. 7a). On the other hand, depleted metabolic activities are mostly associated with diverse unclassified species of Lachnospiraceae. Probiotic strains were also negatively associated with enterobactin biosynthesis and vitamin B12 biosynthesis (Fig. 7b). As it has been intensively discussed, there are some possibilities that multi-strain of probiotics may less benefit in comparison to the single-strain probiotics [49]. However, it should be noted that this disadvantage was not observed in synbiotic treatment while maintaining the beneficial effects of probiotics treatment.

\section{Supplementary Information}

The online version contains supplementary material available at https://doi. org/10.1186/s13765-020-00572-4.

Additional file 1: Figure S1. Schematic representation of the experimental design.

Additional file 2: Figure S2. Ranges of Good's coverage obtained in this study.

Additional file 3: Figure S3. Microbial composition at the phylum (A), family (B) and genus (C).

\section{Acknowledgements}

This work was carried out with the support of the "Cooperative Research Program for Agriculture Science and Technology Development (Project No. PJ01331801)" Rural Development Administration, Republic of Korea. This research was partly supported by Korea Basic Science Institute (National research Facilities and Equipment Center) grant funded by the Ministry of Education (Grant No. 2020R1A6C101A188).

\section{Authors' contributions}

Conceptualization: TU and MC; formal analysis: VS, KM and $\mathrm{CH}$; funding acquisition: TU and MC; project administration: YMK; resources: SHK and JL; supervision: SP, TU and MC; writing - original draft: VS, KM, TU and MC. All authors read and approved the final manuscript.

Availability of data and materials

Not applicable.

\section{Competing interests}

The authors declare that they have no competing interests.

\section{Author details}

${ }^{1}$ Faculty of Biotechnology, School of Life Sciences, SARI, Jeju National University, Jeju 63243, Republic of Korea. ${ }^{2}$ Department of Biochemistry, School of Medicine, Jeju National University, Jeju 63243, Republic of Korea. ${ }^{3}$ Department of Life \& Environmental Science, Daegu University, Daegu 38453, Republic of Korea. ${ }^{4}$ Quegen Biotech Co. Ltd., Seoul, Republic of Korea. ${ }^{5}$ Department of Pathology, School of Medicine, Jeju National University, Jeju 63243, Republic of Korea. ${ }^{6}$ Subtropical/Tropical Organism Gene Bank, Jeju National University, Jeju 63243, Republic of Korea. ${ }^{7}$ Institute of Medical Science, Jeju National University, Jeju 63241, Republic of Korea. ${ }^{8}$ Interdisciplinary Graduate Program in Advanced Convergence Technology \& Science, Jeju National University, Jeju 63241, Republic of Korea.

Received: 5 October 2020 Accepted: 28 November 2020 Published online: 17 January 2021

\section{References}

1. Guinane CM, Cotter PD (2013) Role of the gut microbiota in health and chronic gastrointestinal disease: understanding a hidden metabolic organ. Ther Adv Gastroenterol 6(4):295-308 
2. Valdes AM, Walter J, Segal E, Spector TD (2018) Role of the gut microbiota in nutrition and health. BMJ (Online) 361:36-44

3. Cani PD (2018) Human gut microbiome: hopes, threats and promises. Gut 67(9):1716-1725

4. Rivera-Piza A, Lee SJ (2020) Effects of dietary fibers and prebiotics in adiposity regulation via modulation of gut microbiota. Appl Biol Chem 63(1):2. https://doi.org/10.1186/s13765-019-0482-9

5. Saulnier DM, Ringel Y, Heyman MB, Foster JA, Bercik P, Shulman RJ et al (2013) The intestinal microbiome, probiotics and prebiotics in neurogastroenterology. Gut Microbes 4(1):17-27

6. Goodrich J, Waters J, Poole A, Sutter J, Koren O, Blekhman R et al (2014) Human genetics shape the gut microbiome. Cell 159(4):789-799

7. Floch MH (2018) The role of prebiotics and probiotics in gastrointestinal disease. Gastroenterol Clin North Am 47(1):179-191

8. Kok CR, Quintero DFG, Niyirora C, Rose D, Li A, Hutkins R (2019) An in vitro enrichment strategy for formulating synergistic synbiotics. Appl Environ Microbiol 85(16):1-15

9. Leeming ER, Johnson AJ, Spector TD, Roy CIL (2019) Effect of diet on the gut microbiota: rethinking intervention duration. Nutrients 11(12):1-28

10. Hwang N, Eom T, Unno T (2018) Comparison of gut microbiome between low fiber and high fat diet fed mice. J Appl Biol Chem 61(2):165-172

11. Shigwedha N, Hiwilepo-van Hal P, Jia L, Sichel L, Zhang S (2016) Prebiotics: metabolism and symbiotic synergy with probiotics in promoting health. Probiotics and prebiotics in human nutrition and health. Rijeka, InTech, pp 76-78

12. Getahun A, Tesfaye A, Muleta D (2016) Investigation of the potential benefits and risks of probiotics and prebiotics and their synergy in fermented foods. Singap J Chem Biol 6(1):1-16

13. Westfall S, Lomis N, Prakash S (2018) A novel polyphenolic prebiotic and probiotic formulation have synergistic effects on the gut microbiota influencing Drosophila melanogaster physiology. Artif Cells Nanomed Biotechnol 46(sup2):441-455. https://doi.org/10.1080/21691 401.2018.1458731

14. Morshedi M, Saghafi-Asl M, Hosseinifard ES (2020) The potential therapeutic effects of the gut microbiome manipulation by synbiotic containing-Lactobacillus plantarum on neuropsychological performance of diabetic rats. J Transl Med 18(1):1-14. https://doi.org/10.1186/s1296 7-019-02169-y

15. Jeong S-Y, Kang S, Hua CS, Ting Z, Park S (2017) Synbiotic effects of B-glucans from cauliflower mushroom and Lactobacillus fermentum on metabolic changes and gut microbiome in estrogen-deficient rats. Genes Nutr 12(1):1-12

16. Ke X, Walker A, Haange SB, Lagkouvardos I, Liu Y, Schmitt-Kopplin P et al (2019) Synbiotic-driven improvement of metabolic disturbances is associated with changes in the gut microbiome in diet-induced obese mice. Mol Metab 22(February):96-109. https://doi.org/10.1016/j.molme t.2019.01.012

17. Schloss PD, Westcott SL, Ryabin T, Hall JR, Hartmann M, Hollister EB et al (2009) Introducing mothur: open-source, platform-independent, community-supported software for describing and comparing microbial communities. Appl Environ Microbiol 75(23):7537-7541

18. Huse SM, Welch DM, Morrison HG, Sogin ML (2010) Ironing out the wrinkles in the rare biosphere through improved OTU clustering. Environ Microbiol 12(7):1889-1898

19. Quast C, Pruesse E, Yilmaz P, Gerken J, Schweer T, Yarza P et al (2013) The SILVA ribosomal RNA gene database project: improved data processing and web-based tools. Nucleic Acids Res 41(D1):590-596

20. Cole JR, Wang Q, Fish JA, Chai B, McGarrell DM, Sun Y et al (2014) Ribosomal database project: data and tools for high throughput rRNA analysis. Nucleic Acids Res 42(D1):633-642

21. Westcott SL, Schloss PD (2017) OptiClust, an improved method for assigning amplicon-based sequence data to operational taxonomic units. mSphere 2(2):1-11

22. Langille MGI, Zaneveld J, Caporaso JG, McDonald D, Knights D, Reyes JA et al (2013) Predictive functional profiling of microbial communities using 16SrRNA marker gene sequences. Nat Biotechnol 31(9):814-821. https://doi.org/10.1038/nbt.2676
23. Langille MGI (2018) Exploring linkages between taxonomic and functional profiles of the human microbiome. mSystems 3(2):e00163-17

24. Segata N, Izard J, Waldron L, Gevers D, Miropolsky L, Garrett WS et al (2011) Metagenomic biomarker discovery and explanation. Genome Biol 12(6): 1-8

25. Fernandes AD, Reid JNS, Macklaim JM, McMurrough TA, Edgell DR, Gloor GB (2014) Unifying the analysis of high-throughput sequencing datasets: characterizing RNA-seq, 16SrRNA gene sequencing and selective growth experiments by compositional data analysis. Microbiome 2(1):1-13

26. O'callaghan AA, Corr SC (2019) Establishing boundaries: the relationship that exists between intestinal epithelial cells and gut-dwelling bacteria. Microorganisms 7(12):1-12

27. Xiang I, Zhang Y, Bao D, Cao N, Zhang X, Li P et al (2017) C-Abl regulates gastrointestinal muscularispropria homeostasis via ERKs. Sci Rep $7(1): 1-11$

28. Randall KJ, Turton J, Foster JR (2011) Explant culture of gastrointestinal tissue: a review of methods and applications. Cell Biol Toxicol 27(4):267-284

29. Lam KL, Cheung PCK (2013) Non-digestible long chain beta-glucans as novel prebiotics. Bioact Carbohydr Diet Fibre 2(1):45-64. https://doi. org/10.1016/j.bcdf.2013.09.001

30. Wieërs G, Belkhir L, Enaud R, Leclercq S, Philippart de Foy JM, Dequenne I et al (2020) How probiotics affect the microbiota. Front Cell Infect Microbiol 9(January):454

31. Wosinska L, Cotter PD, O'Sullivan O, Guinane C (2019) The potential impact of probiotics on the gut microbiome of athletes. Nutrients 11(10):2270

32. Sagheddu V, Patrone V, Miragoli F, Puglisi E, Morelli L (2016) Infant early gut colonization by Lachnospiraceae: high frequency of Ruminococcus gnavus. Front Pediatr 4(June):7-12

33. Venegas DP, De La Fuente MK, Landskron G, González MJ, Quera R, Dijkstra G et al (2019) Short chain fatty acids (SCFAs) mediated gut epithelial and immune regulation and its relevance for inflammatory bowel diseases. Front Immunol 10(March):277

34. Hiippala K, Kainulainen V, Suutarinen M, Heini T, Bowers JR, Jasso-Selles D et al (2020) Isolation of anti-inflammatory and epithelium reinforcing bacteroides and Parabacteroides spp. from a healthy fecal donor. Nutrients 12(4):935

35. Zhou Y, Zhi F (2016) Lower level of bacteroides in the gut microbiota is associated with inflammatory bowel disease: a meta-analysis. Biomed Res Int 2016:1-2

36. Russo P, López P, Capozzi V, de Palencia PF, Dueñas MT, Spano G et al (2012) Beta-glucans improve growth, viability and colonization of probiotic microorganisms. Int J Mol Sci 13(5):6026-6039

37. Kim S, Jazwinski MS (2018) The gut microbiota and healthy aging. Gerontology 64(6):513-520. https://doi.org/10.1111/mec.13536.Appli cation

38. Smith MW, Neidhardt FC (1983) 2-Oxoacid dehydrogenase complexes of Escherichia coli: cellular amounts and patterns of synthesis. J Bacteriol 156(1):81-88

39. Lieber CS (2002) S-Adenosyl-L-methionine: its role in the treatment of liver disorders. Am J Clin Nutr 76(5):12418503

40. Davis RAH, Deemer SE, Bergeron JM, Little JT, Warren JL, Fisher G et al (2019) Dietary R,S-1,3-butanedioldiacetoacetate reduces body weight and adiposity in obese mice fed a high-fat diet. FASEB J 33(2):2409-2421

41. Degnan PH, Taga ME, Goodman AL (2014) Vitamin B12 as a modulator of gut microbial ecology. Cell Metab 20(5):769-778

42. Tofalo R, Cocchi S, Suzzi G (2019) Polyamines and gut microbiota. Front Nutr 6(February): $1-5$

43. Gao B, Duan Y, Lang S, Barupal D, Wu T, Valdiviez L et al (2020) Functional microbiomics reveals alterations of the gut microbiome and host co-metabolism in patients with alcoholic hepatitis. Hepatol Commun 4:1-22

44. Raymond KN, Dertz EA, Kim SS (2003) Enterobactin: an archetype for microbial iron transport. Proc Natl Acad Sci USA 100(7):3584-3588 
45. Brown J, O'Callaghan CA, Marshall ASJ, Gilbert RJC, Siebold C, Gordon S et al (2007) Structure of the fungal $\beta$-glucan-binding immune receptor dectin-1: implications for function. Protein Sci 16(6):1042-1052

46. Fan P, Song P, Li L, Huang C, Chen J, Yang W et al (2017) Roles of biogenic amines in intestinal signaling. Curr Protein Pept Sci 18(6):532-540

47. Gerner RR, Klepsch V, Macheiner S, Arnhard K, Adolph TE, Grander C et al (2018) NAD metabolism fuels human and mouse intestinal inflammation. Gut 67(10):1813-1823

48. Rooks MG, Garrett WS (2016) Gut microbiota, metabolites and host immunity. Nat Rev Immunol 16(6):341-352
49. Ouwehand AC, Invernici MM, Furlaneto FAC, Messora MR (2018) Effectiveness of multistrain versus single-strain probiotics current status and recommendations for the future. J Clin Gastroenterol 52:S35-40

\section{Publisher's Note}

Springer Nature remains neutral with regard to jurisdictional claims in published maps and institutional affiliations.

\section{Submit your manuscript to a SpringerOpen ${ }^{\circ}$ journal and benefit from:}

- Convenient online submission

- Rigorous peer review

- Open access: articles freely available online

- High visibility within the field

- Retaining the copyright to your article

Submit your next manuscript at $\boldsymbol{\nabla}$ springeropen.com 